\title{
TRATAMENTO DO DIABETES MELITO GESTACIONAL
}

Carlos Alberto Maganha*, Diana Gertrudes Barenboim Salles Vanni, Maria Augusta Bernardini, Marcelo Zugaib

Trabalho realizado no Setor de Endocrinopatias da Clínica Obstétrica do Hospital das Clínicas da Faculdade de Medicina da Universidade de São Paulo, SP.

RESUMO - Neste artigo, os autores analisam os mais recentes avanços no tratamento do diabetes gestacional, enfatizando pontos importantes na abordagem terapêutica: dieta, exercícios, controle glicêmico, utilização da insulina, assim como a utilização de hipoglicemiantes orais. 0 artigo traz propostas atuais para o trata- mento do diabetes melito gestacional bem como ressaltar suas peculiaridades quanto patologia clínico-obstétrica.

UnITERMOS: Diabetes Gestacional. Tratamento. Insulina. Hipoglicemiantes orais. Exercícios.

\section{INTRODUÇÃO}

O diabetes melito gestacional (DMG) é definido como intolerância à glicose de graus variáveis com início ou primeiro diagnóstico durante o segundo ou terceiro trimestres da gestação'. A reclassificação, entretanto, pode ser feita após o parto, utilizando critérios padronizados para a população não-gestante.

Sua incidência é variável, sendo estimada em $3 \%$ a $8 \%$ das gestantes ${ }^{2,3}$.

Agestação é um estado hiperinsulinêmico caracterizado por uma diminuição da sensibilidade à insulina, parcialmente explicada pela presença de hormônios diabetogênicos, tais como a progesterona, o cortisol, a prolactina e o hormônio lactogênico placentário. Os níveis glicêmicos de jejum tendem a ser mais baixos na gestante, contudo, os valores pós-prandiais são mais altos, sobretudo naquelas em que não há aumento adequado da liberação de insulina. As pacientes com DMG apresentam uma diminuição ainda mais acentuada da sensibilidade periférica à insulina, como no diabetes tipo 2, além de uma secreção diminuída de insulina, explicando os picos pós-prandiais. Entretanto, a fisiopatologia do DMG não está totalmente elucidada. Se todas as gestantes fossem resistentes à insulina, a incidência do DMG seria superior aos níveis encontrados.

* Correspondência:

Av. Dr. Enéas de Carvalho Aguiar, 255 -

$10^{\circ}$ andar - Sala 10.083 - Cerqueira César São Paulo - CEP: 05403-000

Telefone: (11) 3069-6209 - Fax: (11) 3081-0115 e-mail: camaganha@uol.com.br
O comprometimento fetal decorre primordialmente da hiperglicemia materna, que por difusão facilitada chega ao feto. A hiperglicemia fetal, por sua vez, estimula a produção exagerada de insulina que interfere na homeostase fetal, desencadeando: macrossomia, fetos grandes para idade gestacional (GIG), aumento das taxas de cesárea, traumas de canal de parto e distocia de ombro, hipoglicemia, hiperbilirrubinemia, hipocalcemia e policitemia fetais, distúrbios respiratórios neonatais e óbito fetal intrauterino ${ }^{4-6}$.

Historicamente, a introdução da insulina na terapêutica da gestante diabética foi um marco na qualidade da assistência a essas muIheres. Os dados sobre diabetes e gestação anteriores à utilização clínica da insulina são sombrios, com relato de cerca de $30 \%$ de mortalidade materna durante a gestação e $50 \%$ de óbitos perinatais ${ }^{7}$. Após a aplicação da insulina no controle do DMG, diminuíram significativamente suas complicações perinatais, com grande impacto principalmentena taxa de óbitos fetais.

$\mathrm{O}$ adequado conhecimento das medidas terapêuticas no diabetes gestacional permitenos alcançar a normoglicemia materna, quebrando o ciclo maléfico dessa entidade e, conseqüentemente reduzindo a incidência de efeitos lesivos ao binômio materno-fetal.

\section{Controle metabólico no DMG}

O monitoramento glicêmico feito através da dosagem da glicemia capilar (dextro), pela própria paciente, várias vezes ao dia é considerado o ideal ${ }^{8}$. Os valores armazenados na memória do aparelho refletem o perfil glicêmico verdadeiro?. Recomendações recentes incluem a monitorização da dextro ao menos quatro vezes ao dia (jejum e duas horas após refeições) na paciente com DMG controlado com dieta, e sete vezes ao dia naquela em insulinoterapia ${ }^{10}$.

Em trabalho realizado por Langer et al." , comparou-se um grupo realizando controle glicêmico sete vezes ao dia (com dextro em aparelho com memória) e outro grupo realizando quatro vezes ao dia (medida estimada da glicemia por fitas-teste), sendo concluído que o grupo com controle mais rigoroso, apresentou menores taxas de cesárea, macrossomia, distocia de ombro e hipoglicemia neonatal. De forma complementar, também pode-se avaliar semanalmente a glicemia plasmática de jejum, a presença de cetonúria e, com menor rigor, a hemoglobina glicosilada ${ }^{12}$.

Segundo recomendações do American Diabetes Association', as glicemias de jejum maiores que $105 \mathrm{mg} / \mathrm{dl}$ e duas horas pós-prandial maiores que $120 \mathrm{mg} / \mathrm{dl}$ estão associadas a maior morbidadeneonatal. Logo, o controle glicêmico deve manter-se nessa faixa de normalidade. Já no 4th International Workshop Conference on Gestacional Diabetes Mellitus, os valores de controle sugeridosforam de glicemias: inferiores a $95 \mathrm{mg} / \mathrm{dl}$ no jejum, inferiores a $140 \mathrm{mg} / \mathrm{dl}(\mathrm{lh}$ pós-prandial) e inferiores a $120 \mathrm{mg} / \mathrm{dl}$ ( $2 \mathrm{~h}$ pósprandial) $)^{13}$. Em estudo realizado por Langer ${ }^{12}$, menores taxas de macrossomia e fetos GIG foram obtidas com os seguintes valores de controle sugeridos: glicemia dejejume pré-prandial inferiores a $95 \mathrm{mg} / \mathrm{dl}$, pós-prandial inferior a $115 \mathrm{mg} / \mathrm{dl}$ e glicemia média (auto-monitoramento da glicemia) entre 90 e $100 \mathrm{mg} / \mathrm{dl}$. Em outro estudo, realizado por Landon et al. ${ }^{14}$, concluiu-se que a manutenção da glicemia 
capilar média abaixo de $110 \mathrm{mg} / \mathrm{dl}$ reduz a morbidade perinatal do DMG.

Dados opostos foram obtidos por Simpson e Kast em estudo retrospectivo ${ }^{15}$, não sendo observadas diferenças nos resultados maternos e neonatais entre o grupo de diabéticas gestacionais com controle glicêmico mantido inferior a $142 \mathrm{mg} / \mathrm{dl}$ e o grupo controle.

Ainda há controvérsias na literatura em relação ao período pós-prandial que melhor reflete o controle glicêmico (uma ou duas horas após a refeição). Em estudo prospectivo realizado por Sivan etal. ${ }^{16}$, observou-se que a glicemia de uma hora após o desjejum encontrava-se alterada duas e meia vezes mais que a glicemia medida duas horas após, enquanto que 0 oposto ocorreu em relação ao jantar, sendo que a glicemia duas horas pós-prandial encontrada estava alterada duas vezes mais que a glicemia medida um hora após. Dessa maneira, diferentes horários de medida poderiam ser adotados para melhor controle metabólico.

Cabe ressaltar que o controle intensivo da glicemia materna, associando várias medidas de dextro ao dia com valores de referência de normalidade baixos, é custoso e muitas vezes desprovido de aceitação da paciente. O sucesso acaba recaindo ao apelo da expectativa materna pelo seu concepto. Aluta pela acessibilidade de todas as pacientes ao ideal deve ser constante, da mesma forma que a busca de mecanismos simplificados e eficazes.

\section{Tratamento dietético}

O controle dietético do DMG vem sendo utilizado desde o século XIX, variando de dietas com predomínio de gorduras ( $85 \%$ do total de calorias), até a subnutrição e jejum.

Com a introdução da insulina na terapêutica, a taxa de carboidratos na dieta manteve-se entre $35 \%$ a $65 \%$ das calorias ${ }^{17}$, e objetivou-se restrição calórica e de carboidratos para melhor controle glicêmico. Entretanto, é importante a manutenção da oferta protéica na gestação, tanto para o crescimento fetal adequado, quanto para garantir o aumento da produção de insulina necessário na gestação ${ }^{18}$. Orecomendado pela American Diabetes Association é limitar a ingesta de carboidratos em $40 \%$ do total de calorias diárias ${ }^{8}$.

A percentagem de carboidratos da dieta tem correlação significativa com a glicemia pós-prandial. Para manutenção da glicemia pós-prandial menor que $140 \mathrm{mg} / \mathrm{dl}$, são neces- sárias taxas inferiores a $45 \%$ de carboidratos no café da manhã, inferiores a $55 \%$ no almoço e inferiores a $50 \%$ no jantar. Contudo, para controle ainda melhor (com valor de glicemia pós-prandial menor que $120 \mathrm{mg} / \mathrm{dl}$ ), as percentagens de carboidratos são respectivamente $33 \%, 45 \%$ e $40 \%{ }^{19}$.

Estudos demonstram bons resultados em relação ao crescimento fetal com a restrição calórica baseada no peso da paciente, com 30 $\mathrm{Kcal} / \mathrm{Kg} /$ dia em mulheres com $80 \%$ a I $20 \%$ do peso ideal, $24 \mathrm{kcal} / \mathrm{Kg} / \mathrm{dia}$ em pacientes acima de $120 \%$, e $40 \mathrm{Kcal} / \mathrm{Kg} /$ dia naquelas com menos de $80 \%$ do peso ideal. O total de calorias ingeridas deve ser dividido em várias refeições, sendo recomendado: apenas $10 \%$ do total de calorias no café da manhã, já que neste horário ocorrem os picos de hormônio do crescimento e cortisol, gerando maior hiperglicemia pós-prandial; $60 \%$ divididos entre almoço e jantar; e os $30 \%$ restantes divididos entre dois ou três lanches no decorrer do dia ${ }^{17,20,21}$.

O tempo necessário para observar o efeito da dieta sobre o controle do DMG ainda é questionado. Fatores que predizem o controle eresultados perinatais, também permanecem pouco documentados. Em estudo de coorte realizado por McFarland et al..$^{22}$, observou-se que pacientes com glicemia de jejum inferiores a $95 \mathrm{mg} / \mathrm{dl}$ no teste de tolerância oral à glicose, evoluíram com bom controle glicêmico com dieta em até duas semanas. Já naquelas com glicemia superiores a $95 \mathrm{mg} / \mathrm{dl}$, não se obteve melhora do controle glicêmico com dieta após uma semana de observação.

Nos dados obtidos na literatura, cerca de $40 \%$ a $70 \%$ das pacientes com DMG atingiram um bom controle apenas com medidas dietéticas ${ }^{23}$.

\section{Exercícios}

O exercício no DMG tem como objetivo primordial diminuir a intolerância à glicose através do condicionamento cardiovascular, que gera aumento da ligação e afinidade da insulina ao seu receptor através da diminuição da gordura intra-abdominal, aumento dos transportadores de glicose sensíveis à insulina no músculo, aumento do fluxo sanguíneo em tecidos sensíveis à insulina e redução dos níveis de ácidos graxos livres ${ }^{24}$. Cronicamente, 0 exercício também aumenta o número de transportadores de glicose no músculo
(GLUT4). Além disso, o consumo muscular é responsável pela retirada de $75 \%$ da glicose sangüínea ${ }^{25}$.

A maior preocupação, contudo, é a segurança na gravidez para a mãe e para o feto. Os parâmetros mais importantes a seremavaliados durante a prática esportiva, visando o bem-estar materno-fetal são: freqüência cardíaca, pressão arterial, temperatura e dinâmica uterinamaternas; efreqüência cardíaca fetal ${ }^{26-30}$.

Vários tipos de aparelhos e exercícios foram testados durante a gestação: entre eles, a bicicleta ergométrica vertical, esteira ergométrica, remo, bicicleta ergométrica horizontal (com encosto) e ergométrica para os membros superiores. A bicicleta convencional causou maior propensão a contrações uterinas, assim como a caminhada com passos mais acelerados; os demais exercícios demonstraram-se seguros, sendo aquele em ergométrica de membros superiores o mais satisfatório deles ${ }^{3 !}$.

Observa-se melhora dos controles glicêmicos e da hemoglobina glicosilada, após três semanas com a dieta e programa de exercícios assistidos durante 20 minutos, em três sessões semanais, quando comparadas a gestante apenas com dieta ${ }^{23}$.

Entretanto, os benefícios do exercício físico no controle do diabetes gestacional carecem de estudos mais aprofundados. De fato, em estudo realizado por Bung et al. ${ }^{32}$, não houve diferença significativa em relação a média de valores de glicemia, peso ao nascimento e taxa de fetos macrossômicos.

\section{Insulinoterapia}

Ainsulina foi isolada e inicialmente utilizada nos casos de diabetes em Toronto, por Frederick Banting, Charles Best,J.J. Macleode J.B. Collip, em $1922^{25}$.

Entre os diversos tipos de insulina hoje disponíveis, temos as de curta ação-regulare lispro - e as de ação mais prolongada - intermediária(NPH), lenta e ultralenta.

Ainsulina lispro, análoga de insulina regular humana (com pico de ação atingido dentro de uma hora da injeção), tem-se demonstrado segura durante a gestação. Não há aumento de malformações congênitas e é significativa a melhora do controle glicêmico pós-prandial e da hemoglobina glicosilada em longo prazo, não havendo, no entanto, diferenças em relação à insulina regular quanto aos resultados 
perinatais ${ }^{33}$. Contudo, trabalhos mais sólidos são necessários para sua maior aplicabilidade.

\section{Quando introduzir?}

De maneira geral, a insulina é introduzida quando a dieta e os exercícios não levam a um controle metabólico adequado, critério esse que varia de autor para autor ${ }^{3,12,34-39}$.

Dados na literatura diferem muito quanto à percentagem de pacientes com DMG que requerem uso de insulina, variando entre $30 \%$ e $60 \%{ }^{24}$.

A recomendação do American College of Obstetrics/Gynecology (ACOG) é introduzir a insulina quando, após realizada dieta adequada, a glicemia de jejum estiver superior a $105 \mathrm{mg} /$ dl e/ou pós-prandial (duas horas) superior a $120 \mathrm{mg} / \mathrm{dl}$. Essa metodologia mantém concordância com $\circ 2^{\text {nd }}$ e $3^{\text {rd }}$ International Workshop- Conferences on Gestacional Diabetes Mellitus ${ }^{34-36}$.

Já no $4^{\text {th }}$ International Workshop-Conference, o limite da glicemia de jejum baixou para $90 \mathrm{mg} / \mathrm{dl}$, conforme dados mais consistentes da literatura embasados na diminuição das taxas de macrossomia ${ }^{13}$.

Entretanto, em estudo realizado por Langer $^{12}$, foi observado que no grupo de pacientes com glicemia de jejum entre $96 \mathrm{e}$ $105 \mathrm{mg} / \mathrm{dl}$, tratadas apenas com dieta, houve três vezes mais fetos grandes para a idade gestacional comparado ao mesmo grupo tratado com dieta e insulina.

Segundo Jovanic-Peterson ${ }^{20}$ são critérios para iniciar insulina após terapêutica com dieta sem controle adequado: glicemia plasmática de jejum maior que $90 \mathrm{mg} / \mathrm{dl}$ ou dextro de jejum superior a $80 \mathrm{mg} / \mathrm{dl}$; dextro pós-prandial (uma hora) superior a $140 \mathrm{mg} / \mathrm{dl}$; e/ou cetonúria persistente.

Em trabalho realizado por Hopp ${ }^{38}$, foi utilizada a concentração de insulina intraamniótica $(A F I>10 \mathrm{mU} / \mathrm{ml})$ entre 28 e 32 semanas como base para introdução de insulina, obtendo-se menor morbidade perinatal com este critério em comparação com o critério valor de glicemia média (média de nove valores em 24 horas após uma semana com tratamento dietético).

Outro critério utilizado para introdução de insulina vem sendo a circunferência abdominal fetal entre 29 e 33 semanas de gestação. Em estudo realizado por Buchanan et al. ${ }^{39}$, utilizando como critério para a introdução de insulina a circunferência abdominal maior que o percentil 75 , foram obtidos três vezes menos fetos GIG em relação a grupo com o mesmo critério tratado apenas com dieta. A circunferência abdominal fetal, parâmetro de crescimento de tecido sensível à insulina, ter-se-ia mostrado influenciada pelos picos de glicemia pós-prandial, inclusive em gestantes não-diabéticas, podendo por isso ser considerada mais um parâmetro de controle metabólico ${ }^{40}$.

Autilização dos valores de testes de tolerância oral à glicose como preditores da necessidade de insulina foi estudada por $T a{ }^{41}$, sendo a glicemia determinada após duas horas da sobrecarga aquela com melhor correlação. Porém, nenhum dos três valores obtidos parece ter sensibilidade ou especificidade suficientes.

Alguns autores defendem ainda a introdução sistemática de doses baixas de insulina. Emestudo prospectivorandomizado Thompson et al. ${ }^{42}$, foram introduzidos, aleatoriamente, insulina NPH e regular em doses de $20 \mathrm{U}$ e IOU ao dia, respectivamente. Dessa forma, obtiveram melhores resultados perinatais quanto ao peso fetal quando comparadas a gestantes tratadas com dieta, apesar de controle metabólico semelhante. Observou-se, portanto, que as gestantes tratadas com dieta apenas têm maior incidência de macrossomia e fetos GIG, independentemente dos níveis glicêmicos.

\section{Esquemas terapêuticos}

Vários esquemas e doses vêm sendo sugeridos no tratamento do DMG, porém muitas vezes com resultados discordantes.

A dose inicial de insulina é geralmente calculada de acordo com o peso da paciente e idade gestacional, sendo propostas as seguintes doses: $0,8 \mathrm{U} / \mathrm{Kg} /$ dia entre a $24^{\mathrm{a}}$ e $32^{\mathrm{a}}$ semanas; $0,9 \mathrm{U} / \mathrm{Kg} /$ dia entre a $32^{\mathrm{a}}$ e $36^{\mathrm{a}}$ semanas; e I,OU/Kg/dia entre a $36^{\mathrm{a}}$ e $40^{\mathrm{a}}$ semanas de gestação ${ }^{20}$.

Nas pacientes extremamente obesas, a dose inicial pode variar entre I,5 e $2 \mathrm{U} / \mathrm{Kg} / \mathrm{dia}$ para contrapor a resistência à insulina combinadas pela gravidez e obesidade ${ }^{37}$.

Em trabalho realizado por Langer et al. ${ }^{43}$, a dose média de insulina requerida para se obter a normoglicemia foi de $91 \pm 10 \mathrm{U}$, com maiores doses entre a $24^{\mathrm{a}}$ e $30^{\mathrm{a}}$ semanas, sem diferença na dose de insulina entre obesas e não-obesas.
O esquema terapêutico de insulina também é diverso.

Segundo boletim da $\mathrm{ACOG}^{3}$ é geralmente mais fácil para a paciente iniciar com esquemas simples e adaptar-se progressivamente a esquemas mais complexos quando necessário. Contudo, esquemas fracionados, de maneira geral, atingem melhores resultados perinatais $12,20,25,37$.

Um esquema adotado com freqüência éo proposto por Langer ${ }^{12}$, no qual a dose total de insulina é fracionada em três aplicações ao dia: na manhã composta por insulina NPH (4/9 da dose total) e regular (2/9 da dose total); no pré-jantar composta por insulina regular ( $1 / 6$ da dose total); eàs 22 horas composta por insulina NPH (I/6 da dose total $)^{12}$.

Outro esquema proposto é com a dose total dividida em: 4/9 de NPHe 2/9 de regular pela manhã, $1 / 6$ de NPH pré-almoço e I/6 de regular pré-jantar ${ }^{20}$.

Esquemas com quatro aplicações diárias, mimetizando a secreção pancreática normal também são empregados. A dose total de insulina então é dividida em: $5 / 18$ de NPH e 2/9 de regular antes do café, $1 / 6$ de regular pré-almoço, I/6 de regular pré-jantar e I/6 de $\mathrm{NPH}$ às 22 horas $^{37}$.

Em estudo realizado por Nachum et al. ${ }^{44}$, observou-se melhor controle glicêmico quando utilizado esquema com quatro injeções ao dia, sendo três doses de insulina regular pré-refeições e uma dose de NPH à noite, quando comparado a esquemas com três injeções, sem que houvesse, ainda, aumento dos episódios de hipoglicemia grave e também com menor morbidade perinatal.

Em contrapartida, esquemas de insulina mais conservadores, com uma ou duas doses de insulina NPH ao dia são propostos, como em trabalho retrospectivo realizado por Simpson ${ }^{15}$. Emprega-se uma dose de insulina $\mathrm{NPH}$ de 08 ou 12 U pela manhã e introduz-se nova dose de insulina NPH pela noite, quando as dextros superam $140 \mathrm{mg} / \mathrm{d}$. Dessa forma, os resultados perinatais foram comparáveis a gestações sem patologias. Porém, são necessários estudos prospectivos que encaminhem também a essa conclusão.

De maneira geral, a sinalização da conduta indica esquemas fracionados, visando um controle metabólico ótimo; porém, cabe a busca da simplificação dessa visão, por vezes, muito elitista da nossa realidade. 
As doses de insulina são adequadas de acordo com o controle glicêmico da paciente, aumentando-se a insulina regular quando ocorre hiperglicemia pós-prandial e alterando-se a insulina NPH de acordo com as glicemias de jejum e pré-prandiais ${ }^{20}$.

Os ajustes da insulina de acordo com as glicemias pós-prandiais demonstraram melhor controle glicêmico e diminuição do risco de hipoglicemia neonatal, macrossomia e parto cesárea, em relação aos ajustes realizados pelas glicemias de jejum e pré-prandiais ${ }^{45}$.

\section{Período periparto}

Durante o período periparto as necessidades de insulina estão, via de regra, diminuídas em decorrência do jejum relativo e da maior utilização de glicose.

A insulina, então, é geralmente descontinuada, sendo mantida infusão de glicose constante para aporte calórico, devendo-se monitorizar a dextro a cada duas horas, no período de latência do trabalho de parto e a cada hora na fase ativa, mantendo a glicemia entre 70 e $140 \mathrm{mg} / \mathrm{dl}^{20}$ ou entre 70 a $120 \mathrm{mg} /$ $\mathrm{d}^{25}$. Em casos de indução eletiva ou cesárea, administrar entre $1 / 4$ a $1 / 2$ da dose matinal de $\mathrm{NPH}$, suspender a insulina regular da manhã e - café, manter com aporte glicêmico endovenoso e corrigir alterações de glicemia com pequenas doses de insulina regular conformenecessário ${ }^{25}$.

\section{Hipoglicemiantes orais}

Apesar de terem sido por muito tempo evitados na gestação, os hipoglicemiantes orais estão aparecendo nos últimos anos como opção ao tratamento do DMG. As drogas do grupo das sulfoniluréias foram sempre abolidas da gestação, por diversas entidades, por causar potencialmente hipoglicemia neonatal e anomalias fetais ${ }^{1,46,47}$.

Em trabalho realizado por Hellmuth et al. ${ }^{47}$, no qual se estudou comparativamente um grupo de gestantes tratadas com insulina, sulfuniluréias e metformin, verificou-se aumento significativo da mortalidade perinatal e incidência de pré-eclâmpsia nesteúltimo. São de uso comum atualmente, mudaram este panorama ${ }^{48}$. Entretanto, a utilização de drogas como a gliburida e glipizida, que são de uso comum, atualmente mudaram este panorama $^{48}$. Diversas pesquisas laboratoriais demonstraram que a gliburida não cruza a barreira placentária em quantidades apreciáveis, diferentemente de outras drogas anteriormente utilizadas tais como o metformin ${ }^{48,49}$.

Em trabalho prospectivo e randomizado realizado por Langer ${ }^{48}$, foram tratados dois grupos de pacientes com DMG, um com insulina e outro com gliburida, em doses variando de 2,5 a $20 \mathrm{mg}$ ao dia de acordo com os controles glicêmicos. Foram obtidos os mesmos resultados neonatais em relação à taxa de fetos GIG, macrossomia, distúrbios respiratórios neonatais, hipoglicemia e anomalias fetais. As concentrações de insulina obtidas do cordão foram semelhantes ea gliburida não foi identificada no sangue de cordão de nenhum dos casos.

Trabalhos recentes também abordam a diminuição significativa dos custos da terapêutica com gliburida em relação àquela adotada com insulina, quando a paciente não atinge controle significativo com dieta e exercícios ${ }^{50}$.

Apesar de emergentes, novas terapêuticas merecem cuidadosa análise de seu real valor para o tratamento do DMG.

\section{Conclusões}

O grande impacto da descoberta da insulina e o profundo conhecimento do mecanismo de acometimento fetal pela hiperglicemia materna e das medidas terapêuticas no diabetes gestacional parece-nos de inquestionável importância. Contudo, o padrão ideal de controle glicêmico conseguido pela união de equipes multidisciplinares e aperfeiçoamento tecnológico são custosos e desprovidos de incentivos. Logo, a intensa procura por mecanismos alternativos e acessíveis a toda população são fundamentais e devem ser valorizados.

\section{SUMMARY}

Management of gestational diabetes

The authors go review recent advanes in the treatment of gestational diabetes, giving emphasis to the peculiarities of this both clinical and obstetric disease. The covered topics covered include diet, exercise, metabolic control, insulin therapy and new therapeutic approaches, such as the use of oral hipoglycaemic agents. [Rev Assoc Med Bras 2003; 49(3): 330-4]

KEY WORDS: Gestational diabetes. Management. Insulin. Oral hypoglycaemic agents. Exercises.

\section{RefERÊNCIAS}

I. American Diabetes Association. Clinical practice recommendations 1996. Diabetes Care 1996; I9(Suppl I):SI-II8.

2. Schmidt MI, Matos MC, Reichelt AJ, Forti AC, de Lima L, Duncan BB. Prevalence of gestational diabetes mellitus-do the new WHO criteria make a difference? Brazilian Gestational Diabetes Study Group. Diabetes Med 2000; 17:376-80.

3. ACOG Practice Bulletin. Clinical management guidelines for obstetrician-gynecologists. Number 30, September 200I (replaces Technical Bulletin Number 200, December 1994). Gestational diabetes. Obstet Gynecol 200I; 98:525-38.

4. O'Sullivan JB, Mahan CM, Dandrow RV. Gestational diabetes and perinatal mortality rate. Am J Obstet Gynecol 1 973; I 16:901-04.

5. Langer O. Gestational diabetes is a clinical entity: the cost of non treating. Am J Obstet Gynecol 1997; 175: 186.

6. Pedersen J. The pregnant diabetic and her newborn. Problems and management. In: Hellman LM, Pritchard JA, editors. Williams obstetrics. Baltimore: Springer; 1977.

7. Feudtner C, Gabbe SG. Diabetes and pregnancy: four motifs of modern medical history. Clin Obstet Gynecol 2000; 43:4-16.

8. Standards of medical care for patients with diabetes mellitus. American Diabetes Association. Diabetes Care 1994; 17:61623.

9. Langer O, Langer N, Piper JM, Elliott B, Anyaegbunam $A$. Cultural diversity as a factor in self-monitoring blood glucose in gestational diabetes. J Assoc Acad Minor Phys 1995; 6:73-7.

10. Jovanovic-Peterson L, Peterson CM. Sweet success, but an acid aftertaste? N Engl J Med 1991; 325:959-60.

II. Langer O, Rodriguez DA, Xenakis EM, McFarland MB, Berkus MD, Arrendondo F. Intensified versus conventional management of gestational diabetes. Am J Obstet Gynecol 1994; 170: 1036-46; discussion 1046-7.

12. Langer $O$. Maternal glycemic criteria for insulin therapy in gestational diabetes mellitus. Diabetes Care 1998; 2 I (Suppl 2):B9 I-8.

13. Jovanovic L. American Diabetes Association's Fourth International Workshop-Conference on Gestational Diabetes Mellitus: summary and discussion. Therapeutic interventions. Diabetes Care 1998; 2 I (Suppl 2):B I I -7.

14. Landon MB, Gabbe SG, Piana R, Mennuti MT, Main EK. Neonatal morbidity in pregnancy complicated by diabetes mellitus: predictive value of maternal glycemic profiles. Am J Obstet Gynecol 1 987; 156: 1089-95.

15. Simpson RW, Kast SJ. Management of gestational diabetes with a conservative insulin protocol. Med J Aust 2000; 172:537-40.

16. Sivan E, Weisz B, Homko CJ, Reece EA, Schiff E. One or two hours postprandial glucose measurements: are they the same? Am J Obstet Gynecol 200 I; 1 85:604-7. 
17. Jovanovic-Peterson L, Peterson CM. Dietary manipulation as a primary treatment strategy for pregnancies complicated by diabetes. J Am Coll Nutr 1990; 9:320-5.

18. Ericksson UJ, Sweene I. Diabetes in pregnancy: fetal macrossomia, hyperinsulinism, and islet hyperplasia in the offspring of rats subjected to temporary protein-energy malnutrition early in life. Pediatr Res 1993; 34:791-795.

19. Peterson CM, Jovanovic-Peterson L. Percentage of carbohydrate and glycemic response to breakfast, lunch, and dinner in women with gestational diabetes. Diabetes 1991; 40(Suppl 2): 172-4.

20. Jovanovic-Peterson L, Peterson CM. New strategies for the treatment of gestational diabetes. Ir J Med Sci I99I; 27:5 I0-5.

21. Jovanovic-Peterson L, Druzin M, Peterson CM. The effect of euglycemia in insulindependent diabetic woman. Am J Med I 98I; 71:921-7.

22. McFarland MB, Langer $O$, Conway DL, Berkus $M D$. Dietary therapy for gestational diabetes: how long is long enough? Obstet Gynecol 1999; 93:978-82.

23. Jovanovic-Peterson L, Durak EP, Peterson CM. Randomized trial of diet versus diet plus cardiovascular conditioning on glucose levels in gestational diabetes. Am J Obstet Gynecol 1989; 161:415-9.

24. Langer $O$. Management of gestational diabetes. Clin Obstet Gynecol 2000; 43: 106-I 5.

25. Homko CJ, Khandelwal M. Glucose monitoring and insulin therapy during pregnancy. Obstet Gynecol Clin North Am 1996; 23:47-74.

26. Artal R, Paul RH, Romen Y, Wiswell R. Fetal bradycardia induced by maternal exercise. Lancet 1 984; I:258.

27. Cllings $C A$, Curet $L B$. Fetal heart rate response to maternal exercise. Am J Obstet Gynecol 1985; 151:498.

28. Dressendorfer RH, Gooden RC. Fetal heart rate response to maternal exercise testing. Physician Sport 1980; 8:90-93.

29. ClappJF. Fetal heart rate response to running in midpregnancy and latepregnancy. Am J Obstet Gynecol 1985; 153:25 I.

30. Jovanovic-Peterson L, Kessler A, Peterson
CM. Human maternal and fetal response to graded exercise. J Appl Physiol 1985; 58:17-19.

31. Durak EP, Jovanovic-Peterson L, Peterson CM. Physical and glycemic responses of women with gestational diabetes to a moderately intense exercise program. Diabetes Educ 1990; 16:309-12.

32. Bung P, Bung C, Artal R, Khodiguian N, Fallenstein $F$, Spatling $L$. Therapeutic exercise for insulin-requiring gestational diabetics: effects on the fetus-results of a randomized prospective longitudinal study. J Perinat Med 1993; 21:125-37.

33. Bhattacharyya A, Brown S, Hughes S, Vice PA. Insulin lispro and regular insulin in pregnancy. Q J Med 200I; 94:255-60.

34. ACOG technical bulletin. Diabetes and pregnancy. Number 200_December 1994 (replaces No. 92, May 1986). Committee on Technical Bulletins of the American College of Obstetricians and Gynecologists. Int J Gynaecol Obstet 1995; 48:331-9.

35. Proceedings of the Second International Workshop-Conference on Gestational Diabetes Mellitus. October 25-27, 1984, Chicago, Illinois. Diabetes 1985; 34(Suppl 2): I - 30.

36. Proceedings of the Third International Workshop-Conference on Gestational Diabetes Mellitus. November 8-10, 1990, Chicago, Illinois. Diabetes 1991; 40(Suppl 2): I-20I.

37. Jovanovic L. Role of diet and insulin treatment of diabetes in pregnancy. Clin Obstet Gynecol 2000; 43:46-55.

38. Hopp H, Vollert W, Ragosch V, Weitzel HK, Glockner E, Besch W. Indication and results of insulin therapy for gestational diabetes mellitus. J Perinat Med 1996; 24:52 I -30.

39. Buchanan TA, Kjos SL, Schafer U, Peters RK, Xiang A, Byrne J et al. Utility of fetal measurements in the management of gestational diabetes mellitus. Diabetes Care 1998; 2 I (Suppl 2):B99-106.

40. Parreti E, Mecacci F, Papini M, Cioni R, Carignani L, Mignosa M. Third trimeter glucose levels from diurnal profiles in nondiabetic pregnancies: correlations with sonografic parameters of fetal growth. Diabetes Care 2000; 24:1319-23.
4I. Tan YY, Liauw PC, Yeo GS. Using glucose tolerance test results to predict insulin requirement in women with gestational diabetes. Aust N Z J Obstet Gynaecol 1995; 35:262-6.

42. Thompson DJ, Porter KB, Gunnells DJ, Wagner PC, Spinnato JA. Prophylactic insulin in the management of gestational diabetes. Obstet Gynecol 1 990; 75:960-4.

43. Langer O, Anyaegbunam A, Brustman L, Guidetti D, Levy J, Mazze R. Pregestational diabetes: insulin requirements throughout pregnancy. Am J Obstet Gynecol 1988; 159:616-21.

44. Nachum Z, Ben-Shlomo I, Weiner E, ShalevE. Twice daily versus four times daily insulin dose regimens for diabetes in pregnancy: randomised controlled trial. BMJ 1999; 319:1223-7.

45. Veciana A, Major CA, Morgan MA, Asrat T, Toohey JS, Lien JM et al. Postprandial versus preprandial blood glucose monitoring in women with gestational diabetes mellitus requering insulin therapy. N Engl J Med I 995; 333:1237.

46. Hellmuth E, Damm P, Molsted-Pedersen L. Congenital malformations in offspring of diabetic women treated with oral hypoglycaemic agents during embryogenesis. Diabetes Med 1994; I I:47I-4.

47. Hellmuth E, Damm P, Molsted-Pedersen L. Oral hypoglycaemic agents in 118 diabetic pregnancies. Diabetes Med 2000; I7:507-II.

48. Langer $O$, Conway DL, Berkus MD, Xenakis EM, Gonzales O. A comparison of glyburide and insulin in women with gestational diabetes mellitus. N Engl J Med 2000; 343: I | 34-8.

49. Koren G. Glyburide and fetal safety; transplacental pharmacokinetic considerations. Reprod Toxicol 200I; I 5:227-9.

50. Goetzl L, Wilkins I. Glyburide compared to insulin for the treatment of gestational diabetes mellitus: a cost analysis. J Perinatol 2002; 22:403-6.

Artigo recebido: 16/08/02 Aceito para publicação: 26/02/03 